\title{
Taste alteration, mouth dryness and teeth staining as side effects of medications taken by elderly
}

\author{
Camila Porto de Deco ${ }^{1}$, Michelle Roberta Vieira Silva Reis ${ }^{2}$, Adriana Mathias Pereira da Silva Marchini ${ }^{1}$, \\ Rosilene Fernandes da Rocha ${ }^{1}$, Mateus Bertolini Fernandes dos Santos ${ }^{3}$, Leonardo Marchini ${ }^{4}$
}

${ }^{1}$ Universidade Estadual Paulista - UNESP, São José dos Campos Dental School, Department of Biosciences and Oral Diagnosis, São José dos Campos, SP, Brazil

2Private Practice - São José dos Campos, SP, Brazil

${ }^{3}$ Universidade Federal de Pelotas - UFPEL, School of Dentistry, Department of Restorative Dentistry, Pelotas, RS, Brazil

${ }^{4}$ University of lowa, College of Dentistry, Department of Preventive and Community Dentistry, lowa City, IA, USA

\begin{abstract}
Elderly patients generally use several types of medication, some of which may cause oral side effects. Aim: To investigate the oral side effects caused by medication in an elderly sample. Methods: Three hundred patients were interviewed about their use of medication and were divided in two groups: institutionalized ( $n=150)$ and community-dwelling $(n=150)$ elderly. Results: The most used drugs were antihypertensives (53\%) for community-dwelling elders and antiulceratives (76\%) for the institutionalized ones. The more prevalent side effects were taste alterations that occurred in $19 \%$, dry mouth in $17 \%$ and teeth staining in $2 \%$. Conclusions: A high prevalence of oral side effects from medications used by the elderly was found in this study. The health professionals should be aware of the possible side effects caused by prescribed medications.
\end{abstract}

Keywords: aged; drug interactions; xerostomia.

\section{Introduction}

Received for publication: July 27, 2014 Accepted: November 25, 2014

Correspondence to: Mateus Bertolini Fernandes dos Santos Universidade Federal de Pelotas Faculdade de Odontologia Rua Gonçalves Chaves, 457/502 CEP: 96015-560 Pelotas, RS, Brasil Phone: +55 5399349134 E-mail:mateusbertolini@yahoo.com.br
Aging is characterized by accentuated changes in many organs of the human body $^{1}$. The association of these changes and age-related pathologies usually result in the need for essential medication to maintain the elderly's quality of life. Generally, the elderly use several types of medication ${ }^{2}$. However, the use of multiple medications must be undertaken with caution, as aging also promotes important changes concerning their absorption. Many medications could be dangerous if used together and a great number of medications used by the elderly could also result in an increase of undesirable side effects ${ }^{3}$.

Many undesirable oral side effects may be caused by commonly used medications by the elderly. The most common side effect is mouth dryness (xerostomia $)^{4-5}$. Xerostomia can affect eating, drinking, swallowing and the retention of removable dentures ${ }^{4}$, reducing the elderly's quality of life ${ }^{6}$. Another undesirable oral side effect is taste alteration ${ }^{7}$. Abnormal taste perceptions in the elderly may impair nutritional status and also negatively affect quality of life ${ }^{8}$.

The aim of this study was to investigate which oral side effects could be the result of medication taken by an elderly population sample. Considering the 
results of previous studies ${ }^{4-5}$, it was hypothesized that xerostomia could be the most common oral side effect.

\section{Material and methods}

\section{Sample size}

The sample size for this study was calculated using the Minitab (Minitab Inc., State College, PA, USA) power and sample size tool. Three hundred elderly subjects aged 60 years or over from the elderly residents of São José dos Campos, SP, Brazil, were randomly included and evaluated. This number represented about $1.27 \%$ of the city's elderly population, as described previously in another study ${ }^{2}$. The study sample received a sample power score of 0.94 .

The included patients were divided in two groups: institutionalized $(n=150)$ and community-dwelling $(n=150)$. The use of any medication was not considered as inclusion criterion. This survey was approved by the Research Ethics Committee of the University of Vale do Paraíba (protocol L102/2004/CEP) $)^{2}$.

One single professional visited and interviewed all the patients and the obtained data were recorded on a custommade form, medications were then classified and their possible oral side effects were evaluated by a literature review before being documented. The whole data set was then analyzed statistically.

\section{Statistical Analysis}

The data were evaluated using two ratios equality test, Kruskal-Wallis test, ANOVA, Mann-Whitney test and Spearman's correlation test. All variables (gender, institutionalized or community-dwelling, use and number of medications, pharmacological class and side effects) were compared to check for possible significant differences and/ or correlations. The level of significance adopted in all tests was set at $95 \%$.

\section{Results}

\section{Sample description}

In the sample, $70 \%(n=211)$ were women. The institutionalized group was older than the communitydwelling group (ANOVA, $\mathrm{p}<0.001$ ). The average age of the institutionalized group was 76.69 years, and among the community-dwelling group it was 71.89 years.

\section{Use of Medication}

In this sample, only $52(17.3 \%)$ did not use any medications, which was significantly different from the number of patients that took at least one medication (two ratios equality test; $\mathrm{p}<0.001$ ), The institutionalized elderly used more (KruskalWallis, $p<0.001)$ medication (3.26) than the communitydwelling group (1.97), as seen previously in another study ${ }^{2}$. There was no correlation between age and number of medications used (Spearman's correlation, $\mathrm{p}<0.173$ ).

The most common type of medication used by the elderly were antihypertensive drugs (Table 1), which presented a statistically significant difference to the other medications ( $58 \%$, two ratios equality test, $\mathrm{p}<0.001)$, as observed in Table 2 .

\section{Oral side effects}

Taste alteration was the most frequent oral side effect, occurring in $19 \%$ of the total sample, followed by dry mouth $(17.3 \%)$ and teeth staining $(2.3 \%)$.

Table 3 illustrates the oral side effects, which could be

Table 1 - Pharmacological classification of medication used by an elderly sample of São José dos Campos, SP, Brazil in 2012.

\begin{tabular}{lccc}
\hline & Institutionalized & Community-dwelling & Total \\
Antihypertensives & $47 \%(n=82)$ & $53 \%(n=92)$ & $58 \%(n=174)$ \\
Diuretics & $61 \%(n=42)$ & $39 \%(n=27)$ & $23 \%(n=69)$ \\
NSAIDs* & $50 \%(n=34)$ & $50 \%(n=34)$ & $23 \%(n=68)$ \\
Vitamin supplements & $80 \%(n=52)$ & $20 \%(n=13)$ & $22 \%(n=65)$ \\
Antidiabetics & $55 \%(n=27)$ & $45 \%(n=22)$ & $16 \%(n=49)$ \\
Antiulceratives & $76 \%(n=32)$ & $24 \%(n=10)$ & $14 \%(n=42)$ \\
\hline
\end{tabular}

*NSAIDs= non-steroidal anti-inflammatory drugs

Table 2 - P-values chart comparing the use of the pharmacological classes in the sample (Two ratios equality test).

\begin{tabular}{|c|c|c|c|c|c|}
\hline Pharmacological class & Antihypertensives & Diuretics & NSAIDs* & Vitamin supplements & Antidiabetics \\
\hline Diuretics & $<0.001$ & - & - & - & - \\
\hline NSAIDs* & $<0.001$ & 0.923 & - & - & - \\
\hline Vitamin supplements & $<0.001$ & 0.695 & 0.768 & - & - \\
\hline Antidiabetics & $<0.001$ & 0.040 & 0.050 & 0.096 & - \\
\hline Antiulceratives & $<0.001$ & 0.005 & 0.006 & 0.014 & 0.426 \\
\hline
\end{tabular}

*NSAIDs= non-steroidal anti-inflammatory drugs 
Table 3 - Oral side effects possibly caused by each pharmacological classification group that were reported by the elderly patients.

\begin{tabular}{|c|c|c|c|c|}
\hline & & Taste alterations & Xerostomia & Teeth staining \\
\hline \multirow[t]{2}{*}{ Antihypertensives } & Inst & $57 \%(n=47)$ & $17 \%(n=14)$ & - \\
\hline & $C-D$ & $80 \%(n=74)$ & $9 \%(n=8)$ & - \\
\hline \multirow[t]{2}{*}{ Diuretics } & Inst & - & $12 \%(n=5)$ & - \\
\hline & $C-D$ & - & - & - \\
\hline \multirow[t]{2}{*}{ NSAIDs* } & Inst & - & $3 \%(n=1)$ & - \\
\hline & $C-D$ & - & $5 \%(n=2)$ & - \\
\hline \multirow[t]{2}{*}{ Supplements } & Inst & - & $4 \%(n=2)$ & $29 \%(n=15)$ \\
\hline & $C-D$ & - & $23 \%(n=3)$ & $23 \%(n=15)$ \\
\hline \multirow[t]{2}{*}{ Antidiabetics } & Inst & $18 \%(n=5)$ & - & - \\
\hline & $C-D$ & $4 \%(n=1)$ & - & - \\
\hline \multirow[t]{2}{*}{ Antiulcerants } & Inst & $12 \%(n=4)$ & $3 \%(n=1)$ & \\
\hline & $C-D$ & $10 \%(n=1)$ & $40 \%(n=4)$ & - \\
\hline
\end{tabular}

${ }^{*}$ NSAIDs = non-steroidal anti-inflammatory drugs, Inst. = institutionalized elderly, C-D= communitydwelling elderly.

caused by each medication group and the percentage of elderly who presented the effects. Antihypertensives were the most common type of medication to cause oral side effects.

\section{Discussion}

As previously stated, xerostomia is a common undesirable side effect of polypharmacy among the elderly ${ }^{4-}$ ${ }^{5}$, which negatively affects the quality of $\operatorname{life}^{6}$. In the studied sample, xerostomia was the second most common undesirable oral side effect, after taste alteration.

Thomson et al. ${ }^{9}$, in a longitudinal study with Australian elderly, observed that the prevalence and incidence of xerostomia was strongly associated with medication. They observed that diuretics caused xerostomia more frequently. Also, in this longitudinal study, other medications such as aspirin (on a daily basis), non-steroidal anti-inflammatory drugs (NSAIDs) and antidepressants caused side effects9. In the present sample, it was possible to notice that patients using antihypertensive drugs, diuretics, NSAIDs, antiulcerants and even vitamin supplements reported xerostomia. It is important to state that since the vast majority of the patients did not use only one single medication, it is difficult to conclude what pharmacological class of the medications caused xerostomia as a side effect or if it was a result of polypharmacy.

Pajukoski et al. ${ }^{10}$ compared 175 hospitalized patients with 252 patients from outpatient clinics, in Helsinki. They concluded that the greater the number of medications, the greater the probability of xerostomia. Considering that xerostomia was the second most common oral side effect found and that there was considerable polypharmacy in the sample of this study, the here results presented corroborate them. Furthermore, some authors have observed that physiological age-related changes in salivary glands could have a synergistic effect with polypharmacy for causing $x^{x}$ rostomia ${ }^{4-5}$. The management of the xerostomia condition should include patient education, where the patient receives information about the potential sequelae of hyposalivation, including dental caries, candidiasis and mucosal complications $^{11-12}$. It was also found that the use of palliative treatments for dry mouth, such as rinses, sprays, gels and chewing gums could alleviate the symptoms of xerostomia ${ }^{12}$. However, according to a Cochrane review made by Furness et al. ${ }^{13}$, there is no strong evidence that any topical therapy is effective for relieving the symptoms of dry mouth.

The most common undesirable oral side effect found in this study was abnormal taste perception. This may have negative effects on the elderly's general health as it could impair nutritional status. Indeed, taste alteration can affect physical and mental health, and even survival rates ${ }^{8}$. The results presented here suggest that commonly used antidiabetic and antihypertensive medication may cause taste alterations. Most taste alterations induced by medication may reduce after medicine withdrawal, but this could take up to 4 months $^{8}$. Strategies to reduce the risk of adverse drug events include discontinuing medications, prescribing new medications sparingly, reducing the number of prescriptions and frequent medications reconciliation ${ }^{14}$. However, in some cases, discontinuing treatment or changing the medication is not an option, as some medications are required to be taken for the patient's entire life. Health professionals should be aware observing these changes and to dialogue with the medical staff about possible changes in the medication of patients in order to promote the elderly's well-being and quality of life.

In elderly patients, stains are caused by extrinsic pigmentation of teeth and commonly caused by tea, coffee, red wine, tobacco smoking, food with dyes, and numerous medications, such as chlorhexidine, oral iron salts in liquid form, essential oils and co-amoxiclav ${ }^{15}$. Tooth staining is not considered to be a public health problem. However, it has been reported that drug-induced tooth staining is a common cause of decline in use or withdrawal of certain medications ${ }^{16}$. On the other hand, it is important to state that most of extrinsic stains in teeth may be removed by external cleaning ${ }^{15}$. 
The results of this study suggest that medication used by the elderly may cause a high prevalence of oral side effects, such as taste alterations, dry mouth and also teeth staining. These side effects could compromise feeding, talking, esthetics and even nutritional status. The interpretation of the present results should consider some limitations inherent to this study. It is very important that future studies assess what medications have higher potential to cause oral side effects and/or what combination of medications may cause such problems. Also, the city where the study was conducted is considered to be a well-developed city in Brazil, both economically and socially, which may explain the large number of patients that made use of at least one medication.

\section{References}

1. Tamine K, Ono T, Hori K, Kondoh J, Hamanaka S, Maeda Y. Age-related changes in tongue pressure during swallowing. J Dent Res. 2010; 89: 1097-101.

2. de Deco CP, dos Santos JF, da Cunha V de P, Marchini L. General health of elderly institutionalised and community-dwelling Brazilians. Gerodontology. 2007; 24: 136-42.

3. Fick DM, Cooper JW, Wade WE, Waller JL, Maclean JR, Beers MH. Updating the Beers criteria for potentially inappropriate medication use in older adults: results of a US consensus panel of experts. Arch Intern Med. 2003; 163: 2716-24.

4. Gueiros LA, Soares MS, Leao JC. Impact of ageing and drug consumption on oral health. Gerodontology. 2009; 26: 297-301.

5. Leal SC, Bittar J, Portugal A, Falcao DP, Faber J, Zanotta P. Medication in elderly people: its influence on salivary pattern, signs and symptoms of dry mouth. Gerodontology. 2010; 27: 129-33.

6. Gerdin EW, Einarson S, Jonsson M, Aronsson K, Johansson I. Impact of dry mouth conditions on oral health-related quality of life in older people. Gerodontology. 2005; 22: 219-26.

7. Souza LM, Riera R, Saconato H, Demathe A, Atallah AN. Oral drugs for hypertensive urgencies: systematic review and meta-analysis. Sao Paulo Med J. 2009; 127: 366-72.

8. Schiffman SS. Effects of aging on the human taste system. Ann N Y Acad Sci. 2009; 1170: 725-9.

9. Thomson WM, Chalmers JM, John Spencer A, Slade GD, Carter KD. A longitudinal study of medication exposure and xerostomia among older people. Gerodontology. 2006; 23: 205-13.

10. Pajukoski H, Meurman JH, Halonen P, Sulkava R. Prevalence of subjective dry mouth and burning mouth in hospitalized elderly patients and outpatients in relation to saliva, medication and systemic diseases. Oral Surg Oral Med Oral Pathol Oral Radiol Endod. 2001; 92: 641-9.

11. Ismail AI, Tellez M, Pitts NB, Ekstrand KR, Ricketts D, Longbottom C, et al. Caries management pathways preserve dental tissues and promote oral health. Community Dent Oral Epidemiol. 2013; 41: e12-40.

12. Plemons JM, Al-Hashimi I, Marek CL. Managing xerostomia and salivary gland hypofunction: Executive summary of a report from the American Dental Association Council on Scientific Affairs. J Am Dent Assoc. 2014; 145: 867-73.

13. Furness $\mathrm{S}$, Worthington HV, Bryan G, Birchenough S, McMillan R. Interventions for the management of dry mouth: topical therapies. Cochrane Database Syst Rev. 2011; (12): CD008934.

14. Pretorius RW, Gataric G, Swedlund SK, Miller JR. Reducing the risk of adverse drug events in older adults. Am Fam Physician. 2013; 87: 331-6.

15. Abdollahi M, Rahimi R, Radfar M. Current opinion on drug-induced oral reactions: a comprehensive review. J Contemp Dent Pract. 2008; 9: 1-15.

16. Kumar A, Kumar V, Singh J, Hooda A, Dutta S. Drug-induced discoloration of teeth: an updated review. Clin Pediatr (Phila). 2012; 51: 181-5. 